\title{
Direct Transcriptomic Comparison of Xenobiotic Metabolism and Toxicity Pathway Induction of Airway Epithelium Models at an Air Liquid Interface Generated from Induced Pluripotent Stem Cells and Primary Bronchial Epithelial Cells
}

Ivo Djidrovski

Newcastle University Institute of Genetic Medicine

Maria Georgiou

Newcastle University Institute of Genetic Medicine

Elena Tasinato

Newcastle University Institute of Genetic Medicine

Martin O. Leonard

Public Health England

Jelle Van den Bor

Vrije Universiteit Amsterdam

Majlinda Lako

Newcastle University Institute of Genetic Medicine lyle armstrong ( $\square$ lyle.armstrong@ncl.ac.uk)

Newcastle University Institute of Genetic Medicine https://orcid.org/0000-0002-7234-9362

\section{Research Article}

Keywords: Induced pluripotent stem cells, airway epithelium, air-liquid interface, toxicity assessment, transcriptomics

Posted Date: October 29th, 2021

DOI: https://doi.org/10.21203/rs.3.rs-979260/v1

License: (c) (i) This work is licensed under a Creative Commons Attribution 4.0 International License. Read Full License 


\section{Abstract}

The airway epithelium represents the main barrier between inhaled air and the tissues of the respiratory tract and is therefore an important point of contact with xenobiotic substances into the human body. Several studies have recently shown that in vitro models of the airway grown at an air liquid interface (ALI) can be particularly useful to obtain mechanistic information about the toxicity of chemical compounds. However, such methods are not very amenable to high throughput since the primary cells cannot be expanded indefinitely in culture to obtain a sustainable number of cells. Induced pluripotent stem cells (iPSCs) have become a popular option in the recent years for modelling the airways of the lung, but despite progress in the field, such models have so far not been assessed for their ability to metabolise xenobiotic compounds and how they compare to the primary bronchial airway model (pBAE). Here we report a comparative analysis by TempoSeq (oligo directed sequencing) of an iPSC derived airway model (iBAE) with a primary bronchial airway model (pBAE). The iBAE and pBAE were differentiated at an ALI and then evaluated in a 5-compound screen with exposure to a sub lethal concentration of each compound for 24 hours. We found that despite lower expression of xenobiotic metabolism genes, that the iBAE similarly predicted the toxic pathways when compared to the pBAE model. Our results show that iPSC airway models at ALI show promise for inhalation toxicity assessments with further development.

\section{Introduction}

The airway epithelium represents the primary barrier between inhaled air and the tissues of the respiratory tract and is therefore an important point of contact and/or entry of xenobiotic substances into the human body. Such substances may be subject to deliberate introduction required for delivery of therapeutic agents to treat respiratory diseases such as asthma and chronic obstructive pulmonary disease or accidental exposure such as entry of environmental pollutants, industrial chemicals, or household products. There is a need to assess the safety of materials entering the respiratory system. In the case of inhaled therapeutics, unacceptable levels of toxicity are a major obstacle to the development of new treatments, this being a major reason for attrition of drug candidates during therapy development which is reported to account for approximately $30 \%$ of inhaled drug project closures [Cook et al. 2014]. A significant problem for the development of inhaled therapeutics is that toxicity is often detected only during pre-clinical in vivo testing in animal models which, although not a late stage of the development pipeline, will still have required considerable expenditure to progress to the point of failure. In view of this, methods to quantify or even predict the toxicity of xenobiotics are valuable tools since they have the potential to eliminate molecules at an earlier stage. Moreover, toxicity data from such models may also improve the design of downstream animal toxicity studies thereby contributing to animal welfare in accord with the "3Rs" principles of replacement, reduction, or refinement. Although animal models are considered a "gold standard" for toxicity assessment there is a drive to replace as many of these as possible since, quite apart from obvious ethical issues, the response, or animals to drugs or other xenobiotics is not always a close parallel of the human response to the same materials. There are now 
global efforts underway to develop in vitro and in silico methods which can reliably predict the toxicity of chemicals, particularly by the EU with Directive 2010/63/ [Sykes et al. 20212; Olsson et al. 2010], however there is also a pressing need to develop effective in vitro assays which replicate the response of human tissues as closely as possible.

A small number of Air-Liquid interface (ALI) in vitro cell based models of the human airway are commercially available such as Mucilair ${ }^{\mathrm{TM}}$ (https://www.epithelix.com) and Epi-AirwayTM (https://www.mattek.com) and several of these have been evaluated both for assessment of toxicity of environmental toxins such as cigarette smoke, transport vehicle exhaust and industrial toxins and limited studies of inhaled drug toxicity [Sexton et al 2008; Huang et al. 2013]. These types of models are constructed via culture of primary human airway epithelial cells at an ALI whereupon they generate a pseudostratified airway epithelium composed of secretory, ciliated, and basal cells [De Jong et al. 1994] that shows a degree of structural and transcriptomic similarity to the in vivo airway epithelium [Pezzulo et al. 2011; Aufderheide et al. 2015]. Despite the utility of models such as, there are several drawbacks associated with the use of primary airway epithelial cells. Limited availability of cell donors is problematic, but the limited proliferation lifespan of these cells in vitro requires repeated collection of donor tissues. In addition, genetic differences between the donors as well as lifestyle factors (smoking, dietary habits), medical history and donor age can directly impact the results of responses to toxic compounds. Recently, several methods to generate iPSC derived airway cells have been documented in the literature, however none of the studies has examined the expression levels of genes involved in major toxicology pathways and xenobiotic metabolism. Lack of sufficient characterisation prevents widespread application of iPSC models and their development for toxicological purposes. In view of this we have developed an airway epithelial model generated from Induced Pluripotent Stem cells (the iBAE model) at an ALI and directly compared this in parallel to a primary ALI model made from HBECs. We exposed the models to 5 chemicals (Amiodarone, Busulfan, Cerium nanoparticles, paraquat dichloride and Benzo(a)pyrene) at concentrations which do not result in cell death (referred to as sub-lethal concentration hereafter) to understand the induction of toxicity pathways and their effect on expression of xenobiotic metabolism enzymes and transporters which has particular relevance in mechanistic toxicology. To measure the changes of gene expression we used TempoSeq (Templated Oligo assay with Sequencing readout) (http://biospyder.com/) which is a commercial platform focussed on mRNAs representing the major biological and toxicological networks. This comprises the s1500 gene set which identifies sentinel genes from known canonical pathways, which accurately predict pathway perturbations after chemical exposure [Mav et al. 2018] This is a cost-effective approach using RNA from cellular lysates removing the need to extract the RNA from the sample [House et al. 2017; Bushel et al. 2018]. RNA-Seq has been employed for several studies of xenobiotic metabolism in the lung in vivo compared to in vitro models by looking at the expression of different xenobiotic metabolising enzymes, transporters, and receptors [Boei et al. 2017; Courcot et al. 2012].

Transcriptomic analysis may also be used for toxicological assessment of xenobiotic materials by quantitation of differentially expressed genes involved in specific gene regulatory networks or signal 
transduction mechanisms. Such genes may be grouped in so-called pathways of toxicity based upon the transcriptional response of genes in that pathway to specific xenobiotic materials. Several pathways have been described (https://www.wikipathways.org/). For example, XBP1 binds the sequence CCACG in a wide number of genes which respond to endoplasmic reticulum stress [Acosta-Alvear et al. 2007] and contributes to the unfolded protein response mechanism [Lee et al. 2003] of which ATF4 is also a part. The latter gene can also activate other components of the endoplasmic reticulum (ER) stress mechanism such as C/EBP homologous protein transcription factor (CHOP), which engages downstream targets to initiate apoptosis in adverse conditions such as microbial infection [Hu et al. 2019]. ATF4 has roles also in control of translation initiation and stress induced gene expression [Harding et al. 2000; B'Chir et al. 2013] and is probably the best characterised effector in the signalling pathway referred to as the integrated stress response [Pakos-Zebrucka et al. 2016]. Response to electrophilic and oxidising compounds is provided by the KEAP1-NRF2 antioxidant response element system [Kensler et al. 2007], which upregulates expression of antioxidant defence genes [Prestera et al. 1995; Moinova et al. 1999], multidrug response transport proteins [Hayashi et al. 2003], inhibitors of inflammation [Primiano et al. 1998] and initiates replacement of damaged proteins [Kwak et al. 2003]. The aryl hydrocarbon receptor (AhR) is a transcription factor (part of the group of nuclear receptors) that responds to the presence of aromatic hydrocarbons by regulating genes involved xenobiotic metabolism via binding to xenobiotic response elements (XREs) in their promoter regions. AHR targets genes of Phase I and Phase II metabolism, such as cytochrome P450 1A1 (CYP1A1), cytochrome P450 1B1 (CYP1B1), NAD (P)H:quinone oxidoreductase I (NQ01) and aldehyde dehydrogenase 3 (ALHD3A1) [Gassmann et al 2010]. Other mechanisms involve the well characterised p53 DNA damage response [Harris et al. 2005], the NF-KB pathway [Lawrence, L. 2009; Hayden, MS. 2008], regulation of energy metabolism by PPARG/ HIF1a and pathways resulting in activation of transcription factors such as STAT1. Understanding the induction of toxicity pathways can be used for mechanistic toxicology and is of particular importance for making safety assessment of drugs and chemicals.

The results of the transcriptomic analyses used in this study underline the similarities between human airway constructs generated by exposure of both iPSC derived and ex vivo basal cells to an air-liquid interface. In particular, the expression of genes involved in metabolic transformation of xenobiotic substances and the activation of toxic response pathways with sub-lethal chemical concentrations. Our study shows that iPSC models for the modelling of lung airways at ALI may be a useful tool to investigate the toxicity of diverse molecules towards the human airway epithelia which demonstrates the potential utility of the iBAE as a tool for drug development.

\section{Materials And Methods}

\section{Differentiation of iPSC to pseudostratified airway epithelium}

Induced pluripotent stem cell line WT1 (SBAD2) [Buskin et al. 2018] was cultured at $37^{\circ} \mathrm{C}+5 \% \mathrm{CO} 2$ on 6 well plates coated with Matrigel ${ }^{\mathrm{TM}}(\mathrm{BD}, 354230)$ in mTeSRTM1 (StemCell Technologies, 85850) with daily 
media replacement. At 80\% confluency, the cells were passaged with Versene EDTA 0.02\% (Lonza, BE17711E) for 5 minutes and transferred at a split ratio of 1:3 into fresh Matrigel-coated plates. The cells were passaged at least twice before initiating differentiation. Differentiation into airway basal cells involved transit through definitive endoderm and anterior foregut endoderm stages as reported in our previous publication [Djidrovksi et al. 2021]. To isolate basal airway like cells, the day 14 differentiated cells were washed with PBS and enzymatically detached with trypsin for 5 minutes. The detached cells were centrifuged at $300 \mathrm{~g}$ and resuspended in BEGM medium (Lonza, CC-4175) supplemented with $10 \mu \mathrm{M}$ of $\mathrm{Y}$ 27632. They were plated at a ratio of 1 well into 6 mitotically inactivated 3T3 cells. The medium was changed every other day, until $90 \%$ confluency of basal cells is reached. The basal cells can be passaged at a ratio of 7000 cells/ $\mathrm{cm} 2$ on irradiated $3 T 3 \mathrm{~s}$ for at least 2 passages. After reaching $90 \%$ confluency basal cells were harvested as a single cell population by trypsinisation then seeded at a density of 150,000 cells per well onto the apical face of 24 well plate cell culture inserts (ThinCerts ${ }^{\text {TM }}$, Greiner bio-one, 662610 ) with a transparent membrane (PET), with a pore diameter $0.4 \mu \mathrm{m}$, pre-coated with Matrigel $(1: 100)$ and fibronectin (1:100) (Sigma Aldrich, F1141). The adherent cells were fed for three days apically and basolaterally with BEGM medium until they formed a confluent monolayer. Once confluent, the apical medium was removed, and the cells were fed PneumaCult ${ }^{\mathrm{TM}}$ (Stem Cells Technologies, 05001) supplemented with heparin, hydrocortisone and Pen/Strep from the basal chamber. The cells were cultured for 21 days with medium change every other day from the basal chamber.

\section{Differentiation of primary human airway basal cells to pseudostratified epithelium. Human Bronchial} Epithelial cells (HBECs from upper airways, obtained from Dr Martin Leonard) from a single donor were thawed in a petri dish to allow colonies to grow in BEGM media. At $70 \%$ confluency, they were transferred into T75 flasks containing 3T3-J2 cells inactivated by X-irradiation and cultured with BEGM and Rock inhibitor. Similarly, to iPSC derived basal cells, iBCs at $90 \%$ confluency on mitotically inactivated 3T3 feeders were harvested as a single cell population by trypsinisation then seeded at a density of 150,000 cells per well onto the apical face of 24 well plate cell culture inserts (ThinCerts ${ }^{\mathrm{TM}}$, Greiner bio-one, 662610) with a transparent membrane (PET), with a pore diameter $0.4 \mu \mathrm{m}$, pre-coated with Matrigel $(1: 100)$ and fibronectin (1:100) (Sigma Aldrich, F1141). The adherent cells were fed for three days apically and basolaterally with BEGM medium until they formed a confluent monolayer. Once confluent, the apical medium was removed, and the cells were fed PneumaCult ${ }^{\text {tM }}$ (Stem Cells Technologies, 05001) supplemented with heparin, hydrocortisone and Pen/Strep from the basal chamber. The cells were cultured for 21 days and fed every other day from the basal chamber.

\section{Characterisation Of Pseudostratified Epithelia}

Formation of an epithelial barrier was assessed by transepithelial resistance (TEER) as follows: Culture medium was replaced with fresh medium $(500 \mu \mathrm{l} /$ basolateral, $200 \mu \mathrm{l}$ apical) equilibrated to ambient temperature $\left(25^{\circ} \mathrm{C}\right)$. Resistance of the pseudostratified epithelium was measured using an $\mathrm{EVOM} 2^{\mathrm{TM}}$ epithelial voltmeter and compared to resistance of the transwell insert membrane in identical volumes of media but without the presence of cells. To obtain the TEER value in $\Omega . \mathrm{cm}^{2}$, the blank (325 $\Omega$ ) is 
subtracted from the measurement, and then multiplied by 0.334 (which is the area in $\mathrm{cm}^{2}$ of the membrane insert). A TEER value of above $300 \Omega . \mathrm{cm}^{2}$ is an indication of tight junction formation.

Airway constructs matured for 21 days were fixed directly on the membrane with $4 \%$ paraformaldehyde for 10 minutes at $3^{\circ} \mathrm{C}$ and then washed with PBS $(3 \times 1.0 \mathrm{ml})$. The tissues were then removed together with the membrane, placed into moulds, and embedded in Optical coherence tomography (OCT) matrix (Cell Path, KMA-0100-00A). The moulds were placed at $-20^{\circ} \mathrm{C}$ to solidify. Once solid they were sectioned into $5-10 \mu \mathrm{m}$ slices on slides using a cryostat. The sectioned membrane was removed with PBS washes and the slides were then stained using antibodies listed in Table 1.

Table 1

Antibodies used for IHC analysis of iBAE and pBAE constructs

\begin{tabular}{|lllll|}
\hline Antibody name & Dilution & Species & Reference & Supplier \\
\hline CC10 & $1: 100$ & Mouse & sc-365992 & Santa Cruz \\
\hline Synaptophysin & $1: 200$ & Rabbit & YE269 & Abcam \\
\hline ZO-1 & $1: 300$ & Rabbit & $61-7300$ & Invitrogen \\
\hline Acetylated Tubulin & $1: 400$ & Mouse & T6793 & Sigma-Aldrich \\
\hline a-Mouse-AlexaFluor-488 & $1: 1000$ & Goat & A11001 & Invitrogen \\
\hline a-Rabbit-AlexaFluor-647 & $1: 1000$ & Goat & A21245 & Invitrogen \\
\hline
\end{tabular}

Expression of genes specific to the human airway epithelia with a specific focus on cytochrome P450 enzymes was performed using quantitative RT-PCR. Primers employed for this purpose are listed in Table 2.

\section{Analysis of exposure of pseudostratified airway epithelial constructs to xenobiotic materials}

The following chemicals were added to the basal media below iBAE and pBAE airway epithelial constructs; Busulfan $(500 \mu \mathrm{M})$, Paraquat dichloride $(100 \mu \mathrm{M})$ and Amiodarone $(15 \mu \mathrm{M})$. Benzo(a)pyrene $(500 \mu \mathrm{g} / \mathrm{ml})$ and Cerium nanoparticles $(25 \mu \mathrm{g} / \mathrm{ml})(10 \mu \mathrm{l}$ aliquots) were added to the apical face of the constructs. All treated constructs were incubated in contact with the xenobiotic substances ( 24 hours, $37^{\circ} \mathrm{C}, 5 \% \mathrm{CO}_{2}$ ) followed by quantification of TEER. Cytotoxicity was determined using the CyQUANT LDH Cytotoxicity Assay (Cat. Nos. C20300), which includes a positive control of exposure of the airway epithelial constructs to culture media plus $0.1 \%$ triton-X to ensure $100 \%$ cell death after 24 hours exposure.

Airway epithelial constructs treated with xenobiotics as described above were subjected to transcriptomic analysis to compare the similarities of the iBAE model to the pBAE model and to quantify expression changes of genes involved in xenobiotic response and metabolism. Read count data obtained from TempoSeq ${ }^{\mathrm{TM}}$ were first normalised by scaling and subjected to quality control by plotting log transformed bar charts of the normalised count data to detect outliers in the samples. The normalised read counts 
were analysed using DESeq2 [Love et al. 2014] to generate differentially expressed genes, calculating fold changes, $p$-values, base mean, and adjusted $p$-values. The default Wald statistics test was used for significance evaluation. The cut-off of 0.05 was set for adjusted $p$-values and $>2$ or $<-2$ for log 2 fold changes for initial selection of the most significantly differentially expressed genes for the clustering of temporal profiles, development of the profile fitting procedure described below and identification of enriched pathways. The response of $\mathrm{iBAE}$ and $\mathrm{pBAE}$ to xenobiotics was subjected to principal component analysis as follows; the log transformed and scaled TempoSeq count data was used for PCA (prcomp) using R 4.0. The PCA was visualized using the factoextra package.

Table 2. Sequences of primers used for quantification of human airway epithelial genes by Q-RT-PCR 


\begin{tabular}{|ll|}
\hline Sequence name & Sequence \\
\hline nGAPDH forward & GGTTTACATGTTCCAATATGATTCCA \\
\hline nGAPDH reverse & ATGGGATTTCCATTGATGACAAG \\
\hline MUC5AC forward & GCACCAACGACAGGAAGGATGAG \\
\hline MUC5AC reverse & CACGTTCCAGAGCCGGACAT \\
\hline FOXJ1 forward & GGCATAAGCGCAAACAGCCG \\
\hline FOXJ1 reverse & TCGAAGATGGCCTCCCAGTCAAA \\
\hline CC10 forward & TCATGGACACACCCTCCAGTTATGAG \\
\hline CC10 reverse & TGAGCTTAATGATGCTTTCTCTGGGC \\
\hline ASCL1 forward & CCCAAGCAAGTCAAGCGACA \\
\hline ASCL1 reverse & AAGCCGCTGAAGTTGAGCC \\
\hline CYP2F1 forward & ACCCTCCTTAACACCGTCCA \\
\hline CYP2F1 reverse & ATGGCGGTGAGGTACAGAAAG \\
\hline CYP2B6 forward & GCACTCCTCACAGGACTCTTG \\
\hline CYP2B6 reverse & CCCAGGTGTACCGTGAAGAC \\
\hline CYP2J2 forward & GAGCTTAGAGGAACGCATTCAG \\
\hline CYP2J2 reverse & GAAATGAGGGTCAAAAGGCTGT \\
\hline CYP2A6 forward & CGAGACCGTCAGCACCA \\
\hline CYP2A6 reverse & GGATCACTGCCTCCATGT \\
\hline CYP2S1 forward & CGCTACCACTGCTGGGAAACCT \\
\hline CYP2S1 reverse & AATGGGCGTCCTTCTGTCCCC \\
\hline CYP2E1 forward & CCTCCTGCTGGTGTCCATGT \\
\hline CYP2E1 reverse & CTTGGGCTTGGGTCTTCCTGAGTGCT \\
\hline
\end{tabular}

\section{Results}

\section{Airway epithelial basal cells generate pseudostratified airway epithelia at an air liquid interface}


To induce airway epithelial differentiation from iPSC derived and ex vivo primary basal cells, the latter were allowed to grow to confluency on 3T3-J2 feeder layers then harvested as a single cell population by trypsinisation after removal of the bulk of the feeder cells by exposure to $0.48 \mathrm{mM}$ sodium EDTA (Versene, Gibco). The single cells were plated on a transwell insert (ThinCerts ${ }^{\mathrm{TM}}$, Greiner bio-one, 662610), left to grow to confluency and then they were fed from the basal side leaving the apical side in contact with the air. To differentiate the cells into airway epithelia, the commercial PneumaCult ${ }^{\text {TM }}$ media was used with the addition of $10 \mu \mathrm{M}$ DAPT between differentiation days 10 and 14 as shown in the schematic diagram (Figure 1A). Structures with mucous layers present on the apical surface were derived after this differentiation period (Figure 1B) and sections of these structures indicate the presence of a pseudostratified epithelium (Figure 1C). Differentiation of the basal cells into the other cell types present in the pseudostratified epithelium is indicated by the presence of club cell protein 10 (club cells, Figure 1C), and expression of MUC5AC (goblet cells, Figure 1C). The presence of putative pulmonary neuroendocrine cells is indicated by expression of synaptophysin (Figure 1C). In addition, the apical surface of the pseudostratified epithelium comprises ciliated epithelial cells capable of forming tight junction indicated by the presence of ZO-1 and cilia indicated by the presence of acetylated tubulin (Figure 1D). The ability to form tight junctions between the ciliated epithelial cells probably contributes to the TEER values in the range of $250-550 \Omega / \mathrm{cm}^{2}$ by day 60 of culture indicating establishment of an epithelial barrier. The expression of additional markers of airway phenotype was quantified by qRT-PCR showing expression of cytochrome P450 enzymes characteristic of the upper airway. In some instances (CYP2J2, CYP2S1, MUC5AC) expression levels were comparable between the iBAE and pBAE airway constructs, but for most genes (FOXJ1, CC10, CYP2A6, CYP2F1, CYP2B6) expression was lower in iBAE constructs (Figure 1E). Expression of the pulmonary neuroendocrine cell marker ASCL 1 was higher.

Detectable expression of cytochrome P450 enzymes suggests that the iBAE airway epithelial construct might be capable of metabolism parallel to primary basal cell derived constructs albeit, perhaps at lower levels of enzyme activity. In view of this we proceeded to analyse the transcriptomic response of both types of construct to xenobiotic compounds using TempoSeq analysis.

\section{iPSC derived and ex vivo primary basal cell derived airway constructs show comparable transcriptomes with respect to genes included in TempoSeq analysis}

Temposeq ${ }^{\mathrm{TM}}$ transcriptome data were analysed using the Deseq2 package. To obtain log2fold change, TempoSeqR analysis software was used to generate the log2fold differences. The similarity between samples and the heatmaps were generated by Morpheus, (https://software.broadinstitute.org/morpheus). The normalised count comparison of xenobiotic metabolism was graphed using Prism GraphPad. Heatmaps of log2fold changes were generated using R package Pheatmap. Using principal component analysis (PCA), we observed that the transcriptomes of the iBAE constructs cluster together with those of the pBAE construct model and moreover, have the lowest variance between each other when compared with PCA analysis of other iPSC derived cell types produced by the IN3 consortium under which our current project was performed (Figure 2A). We then compared the normalised values between the transcriptome data of undifferentiated iPSCs, iBAE and pBAE constructs and analysed the samples using 
Pearson Correlation (Figure 2B). This indicated low variability between samples (between 0.93 and 1 ) and similarity between the $i B A E$ and $p B A E$ models $(R=0.69 \neg 0.76)$. Comparison of these models was extended to 60 highest expressed genes present in both models; most of the top 60 highest expressing genes in the iBAE model (Figure 2C) are also highly expressed in the pBAE model except for Mt-ND6, CXCL14, TP53/3, EIF4G1 and SLC3A2, which are expressed either at lower levels or not at all in the pBAE model. Conversely, four genes (NEAT1, HSP9OAA1, EPHX1, TPPP3) are expressed in the PBAE model that are not expressed by iBAE constructs. In both models we can find highly expressed keratins (KRT6A, KRT15, KRT17) and annexins ( $A N X A 2 P 2, A N X A 1)$, which are characteristic of lung tissue. Mucin 1 (MUC1) was also found among the top 50 genes expressed in the primary basal cell model and is also highly expressed in the iBAE model. Other common genes that come up in both lists are the eukaryotic translation initiation factors (EIF1, EIF3E) and S100 Calcium Binding Protein P (S100P). The similarities observed in both models and expression of lung specific genes suggests that iPSC differentiation was successful to obtain airway epithelium, however there are still differences in terms of gene expression which could affect the metabolism and toxic response to xenobiotic chemicals.

\section{Expressions of nuclear receptors and phase I/II metabolic enzymes in iPSC derived and ex vivo primary basal cell derived airway constructs}

Organisms have developed a general strategy to protect themselves from possible toxic effects of xenobiotic molecules, which comprises two groups of molecular systems. The first line of defence involves nuclear receptors or xenosensors to detect the presence of potentially harmful materials and xenobiotic metabolizing and transporter systems to break them down to substances that are more easily eliminated. We interrogated TempoSeq ${ }^{\mathrm{TM}}$ data to compare expression levels of key genes in these mechanisms. The aryl hydrocarbon receptor (encoded by $A H R$ ) which normally resides as an inactive complex with heat shock protein $9 \mathrm{p}$ chaperone protein and responds to the presence of planar aromatic hydrocarbons by translocation of the receptor protein to the nucleus where it can activate expression of xenobiotic metabolism genes [Gottlicher M. 1999]. The translocation requires dimerization between the AHR protein and the gene product of $A R N T$, so it is encouraging that these two genes in addition to the feedback repressor $A H R R$ are expressed in iBAE constructs (Figure $3 \mathrm{~A}$ ). Interestingly, $A H R R$ is not detected in the primary basal cell derived constructs. Genes encoding the functionally similar Estrogen receptor ESR1 are present in both pBAE and iBAE constructs while ESR2 is detectable only in the latter. Several members of the nuclear receptor superfamily are expressed at similar levels in both models while three members of the peroxisome proliferator activated group of nuclear receptors (PPARA, PPARG and $P P A R G C 1 A)$, retinoic acid receptor alpha (RARA), thyroid hormone receptor $\beta(T H R B)$ and its interacting protein (TRIP13) and the vitamin D receptor gene (VDR) are also present at comparable levels Together, these data suggest that the iBAE model may be a useful tool for quantifying the response of human airway epithelia to xenobiotic substances.

Phase I metabolism of xenobiotic substances aims to introduce or expose functional groups with the goal of increasing the polarity of the compound and broadly cover oxidation, reduction, and hydrolysis reactions. The iBAE model expresses most of the enzymes involved in phase I metabolism that are 
present on the TempoSeq library (Figure 3B) albeit these are mostly present at lower levels than in pBAE. The first group of genes presented in Figure $3 A$ are members of GDP- glucuronosyltransferase class (UGT1A1, UGT1A10, UGT1A8, UGT2B7). The aldo-keto reductases (AKR1B1, AKR1B10, AKR1C2, AKR1C3, $A K R 7 A 2)$ are also expressed while several enzymes such as UGT1A5, the alcohol dehydrogenases ( $A D H 1 A$ and $A D H 1 B$ ) and $A K R 1 C 2$ were expressed at much higher levels in the pBAE model. The cytochrome P450 enzymes are important contributors to phase I metabolism due to their activity as monoxygenases capable of oxidising diverse xenobiotic substrates [Danielson, PB. 2002]. CYP1A1 is reported to be expressed in human lung [Shimada et al. 1996; Shimada, T. 2006] and to undergo significant induction by exposure to tobacco smoke [Kim et I. 2004] so it is interesting that it is only expressed by the iBAE model (Figure 3C), however the expression of other Cytochrome P450 genes correlates with published reports of their expression in human lung [Carlson, GP. 2008]. High expression of CYP1A1 and CYP4B1 relative to other members of the CYP class is reported in the literature [Gene Set Lung (maayanlab.cloud)] so it is useful to note that the iBAE model demonstrates greater expression levels than the pBAE model, however $C Y P 2 B 7 P$ is the exception being detected only in the primary models. The iBAE model also shows lower expression of CYP2B6, CYP26A1 and CYP2C8 than pBAE but also expresses CYP2W1 which is one of the CYPs expressed in the bronchial mucosa in vivo [Courcot et al 2012]. That notwithstanding, the mRNA expression of phase I enzymes is comparable between the two models

In summary, these data suggest that both $\mathrm{BBAE}$ and pBAE airway models should be capable of phase $\mathrm{I} / \mathrm{II}$ metabolism of xenobiotic molecules.

iPSC derived and ex vivo primary basal cell derived airway constructs show comparable expression of solute transporter protein genes

The solute carrier superfamily comprises over 400 transport proteins responsible for movement of diverse substrates across membranes of the cell or its organelles [Hediger et al 2004]. Several transporter protein genes relevant for toxicological examination are expressed at comparable levels in both airway models (Figure 4A) including neutral amino acid transporters (SLC1A2, SLC1A3, SLC1A4) [Kanai et al. 2013], glucose transporters (SLC2A6, SLC2A1) [Lizak et al. 2019], members of the mitochondrial carrier gene family (SLC25A13, SLC25A14, SLC25A27, SLC25A4, SLC25A46) [Gutierrez-Aquilar, M. 2013] fatty acid transporters (SLC27A1, SLC27A2, SLC27A3) [Anderson et al. 2013], (Figure 4A) with a few notable exceptions. The sulphate ion transporter (SLC26A2) [Heneghan et al. 2010] is absent in the iBAE airway constructs as is choline transporter like protein 4 (SLC44A4) [Nabokina et al. 2015]. Conversely, two glucose transporter proteins ( $S L C 2 A 14$ and $S L C 2 A 3$ ) are absent from the pBAE models although the impact of these discrepancies on the relative metabolic activities in not clear.

\section{Transcriptomic Response Of Airway Constructs To Xenobiotic Molecules}


iPSC and primary basal cell derived models were exposed to five xenobiotic substances with known toxicity against lung /airway tissues at sub-lethal concentrations over 24 hours. Cytotoxicity was assessed by quantifying TEER and LDH although we did not find any significant increase in the LDH activity relative to the untreated control suggesting that the concentrations of chemicals used do not kills cells in the airway constructs. Measurement of TEER before and after treatment indicated that only Amiodarone and Paraquat caused significant reduction in TEER suggesting breakdown of the epithelial barrier (Figure 5A, B). Data obtained from TempoSeq analysis of the transcriptomes of xenobiotic treated cells were processed using the Deseq2 package to generate lists of statistically significant log fold change lists of genes affected by chemical treatment relative to the untreated control [Love et al. 2014] (Figure 6A) and subjected to principal component analyses to quantify the similarity of response to the applied compounds of the iBAE and pBAE models (Figure S1A-E) In performing this analysis, we aimed to show the correlation between the untreated iBAE and pBAE models and between the individual models after treatment with the xenobiotic compounds. The analyses in Figure S1 show that principal components 1 and 2 demonstrate the variance between the iBAE and pBAE models before and after xenobiotic treatment while principal components 3 and 4 account for the variance within the models. These data indicate significant differences between the iBAE and pBAE models, however given the degree of similarity in expression of genes involved in phase 1 and 2 metabolism, we considered the possibility that variance may have arisen from the genetic background of the patient derived cells used to generate the pBAE model and the iPSC used to generate the iBAE model.

We then selected the top 20 upregulated and 10 top downregulated genes with the highest sum of log2fold changes with significantly low $p$-value adjusted $(p<0.05)$. We applied gene ontology analysis to the top 20 genes (up and downregulated), however the limitation of gene ontology analysis is that it is not specific to toxicological processes so we manually annotated the genes identified by TempoSeq that are participants in the major toxicity associated pathways (ATF4 [HU et al. 2019; Lee et al. 2003], Nrf2 [ B'Chir et al. 2013], p53 [Kwak et al. 2003], NFkB [Gassmann et al. 2010; Harris et al. 2005] Ahr [Guerrina et al. 2018], PPARG [Michalik et al. 2006], HIF1a [Masoud et al. 2015], STAT1/2 [Xu et al. 2012], MTF1 [Laity et al. 2007] and XBP1) [Boei et al. 2017] (see Supplementary Table 1 for list of genes assigned to each toxicity pathway). These data were analysed using PathVisio3 [Kutmon et al. 2015] to analyse the log2fold changes of genes induced by the treatment of the chemicals and calculate a Z-score based on the annotation of genes. The pathways are ranked based on their Z-Score in which a positive Z-Score (Z > 1.96) indicates a pathway with more genes meeting the criterion than expected based on the complete dataset. A negative $Z-S c o r e ~(Z<1.96)$ indicates that less genes meet the criterion than expected. An examination of Z-score data suggests that iBAE airway models are marginally more susceptible to toxicological pathway activation than pBAE models (Figure 6B). After application of cerium nanoparticles, the only pathway that shows statistically significant activation in the iBAE model is STAT2 although 87 genes show differential expression between test and control samples. Among the top 20 upregulated genes we can see genes involved several different processes such as apoptotic response (BIRC5, PHLA2), cell cycle arrest and differentiation (INHBA), NFkB inhibition, ciliogenesis (SLC26A)

\section{Supplementary Table 1).}

Page $12 / 27$ 
Amiodarone is a potassium channel blocking agent indicated for life threatening supraventricular and ventricular arrythmias [Baritussio et al 2001] but use of this drug is associated with significant toxic effects against lung tissue, primarily the development of pulmonary fibrosis. As an amphiphilic cationic molecule, amiodarone inhibits lysosomal phospholipases leading to disruption of the lysosome membranes [Haliwell, WH. 1997] and release of reactive oxygen species which may lead to stress pathway activation and lung epithelial cell apoptosis. Surprisingly, our TempoSeqTM analysis of amiodarone exposure did not indicate significant activation of toxicological pathways in either the iBAE or pBAE models. Conversely, significant downregulation of pathways involving NF-kB, $p 53, N R F 2$ and ATF4 is observed in the iBAE model, meaning that other genes outside the pathways are strongly affected. Differentially expressed genes were detected for other GO classes, HAVCR1 (cilia function), $B U B 1$ (apoptosis), CCL4 and PECAM1 (cell adhesion) (Supplementary Table 1).

Busulfan is an alkylating anti-neoplastic agent used to treat chronic myeloid leukaemia [Chen et al. 2013] which can cause interstitial lung fibrosis in humans [Oliner et al. 1961]. In both models Nrf2 was activated (with Z-score 6.9 in iBAE and 4.5 in the pBAE) and p53 (with Z-score 3.2 in iBAE and 2.97 in the pBAE), while NFkB was only affected in the iBAE ( $z$-score=2.07). The most upregulated genes in the iBAE are NMRALP2 (redox sensor), FOSL 1 (involved in cilia), NQO1 (NADP(H)dehydrogenase involved in Nrf2), DNM1, GPX2, CCL4 and BRICS. In the PBAE it as NMRALP2, TRIM16L, GPHT, UHCL 1 (Supplementary Table 1). Activation of $p 53$ and NRF2 is consistent with a response to DNA damage induced by an alkylating agent such as Busulfan.

Paraquat ( $N, N$-dimethyl-4,4'-bipyridinium dichloride) is a redox active heterocyclic compound which functions as an electron transport chain blocking agent. Paraquat has been widely employed to trigger oxidative stress given its unique potency to generate superoxide anion in nearly all experimental systems ranging from isolated mitochondria and mammalian cells [Blanco-Ayala et al. 2014] and as such its mechanism of toxicity is thought to arise by exceeding the capacity of cells to eliminate reactive oxygen species. Several pathways are downstream targets of this imbalance such as NF-KB [Gonzalez-Polo et al. 2004] and UPR [Mottis et al. 2014] so it is interesting to note activation of XBP1, HIF1a, NF-KB, NRF2 and ATF4 in the iBAE model following paraquat exposure (Figure 6B). It noteworthy that NF-KB and HIF1a activation in the pBAE airway model fall short of statistical significance of their z-scores while the p53 DNA damage response which correlates with reported increases of double strand break formation following paraquat exposure in cultured mouse lymphoblasts albeit at a 10x greater concentration than used in our study [Ross et al. 1979]. Differentially expressed genes in the iBAE and pBAE models following Paraquat exposure are shown (Supplementary Table 1).

The polycyclic aromatic hydrocarbon, Benzo(a) pyrene has very similar effects on both models with activation of the NF-KB, NRF2 and aryl hydrocarbon response pathways. The iBAE model shows activation of STAT2 that is on the boundary of statistical significance (Z-score=2.06), which was not seen in the pBAE model while the latter also shows exclusive but modest activation of HIF1a. It is surprising that activation of the DNA damage response, p53 was not observed in either model given the reported ability of Benzo(a)pyrene to induce double strand DNA breaks [Wang et al. 2005], however increased 
activation of AHR, NRF2 and AT4 are consistent with reported toxicity mechanisms of this molecule [Jin et al 2021]. Differentially expressed genes in the iBAE and pBAE models following Benzo(a) pyrene are shown (Supplementary Table 1).

\section{Discussion}

Tissue models derived from iPSC are potentially useful in toxicological assessment of xenobiotic substances. They not only offer unlimited supplies of cells but can also be genetically edited to target specific transporters or enzymes and delineate toxicological pathways. Moreover, they offer the possibility to test the response of cells generated from any individual which may be useful for understanding drug toxicity and efficacy in the pre-clinical phase of drug development. ALI in vitro models of human airways have for toxicity assessment been developed using airway epithelial cells harvested from patients, so the objective of this study was to investigate the potential of iPSC derived in vitro models of the human upper airway for quantification of toxicological responses. ALI constructs similar to the human upper airway epithelium were generated using airway basal cells generated from iPSC derived basal airway cells (iBAE model) and from primary basal cells obtained from a human donor (pBAE model). Both models demonstrate similar morphology and the presence expected cell type however a more detailed characterisation of their transcriptomes was performed using TempoSeq analysis. While this method is useful for determining toxicological responses of a variety of cell types, it is less effective as a tool to confirm differentiation towards an upper airway phenotype since key lung markers genes are not represented in the TempoSeq gene set. For this reason, we characterised our constructs using qPCR, which suggest that the iBAE model is perhaps less mature than the pBAE since there is a lower expression of goblet (MUC5AC), club (CC10) and ciliated marker (FOXJ1) while having a higher expression of neuroendocrine marker ( $A S C L$ 1). Markers of epithelial basal cells (KRT6A, KRT17) and airway epithelium (ANNEXIN1, ANNEXIN2, MUC1, CYP4B) that are present on the TempoSeq gene set are amongst the highest expressed genes detected by this analysis therefore we are confident of the iBAE model's similarity to airway epithelium. Moreover, the top 50 genes detected by TempoSeq analysis of the iBAE and pBAE models are expressed in common indicating a high degree of similarity between the iPSC

and primary basal cell derived models. This is further underlined by the similar expression levels of genes involved in the detection, metabolism, and transport of xenobiotic substances although there are several noteworthy differences. The expression of nuclear receptor genes in iBAE mostly parallels those of $\mathrm{pBAE}$, except ESR1 and $A H R R$, however two genes encoding enzymes which contribute to phase 1 metabolism are expressed only by the iBAE model (CYP11A1, CYP1A1) compared to the pBAE model in which exclusive expression of only $C Y P 2 B 7 p$ is observed. The consequences of these differences are unclear, but it is encouraging that the iBAE model expresses cytochrome P450 genes reported to be preferentially expressed in human lung (CYP1A1, CYP1B1, CYP4B1, CYP2E1 and CYP3A5) [Zhang et al. 2006] at comparable or superior levels to pBAE. Of these genes, CYP1A1 and CYP1B1 show significant induction to xenobiotic materials such as exposure to cigarette smoke [Wiley et al. 1997] whereas CYP2B7P does not despite its high expression level in lung tissue. All other phase 1 metabolism genes show common expression albeit some are present at lower levels in the iBAE model. 
The focus of phase 2 metabolism is the solubilisation of xenobiotic substances or their metabolic products to facilitate elimination. Both the iBAE and pBAE models express a range of phase 2 metabolism genes represented on the TempoSeq gene set, although again there are notable exceptions. None of the genes in this class are exclusive to the pBAE model but $\gamma$-glutamyl transferase and phenylethanolamine N-methyltransferase (GGT1 and PNMT respectively) are only expressed in iBAE. Glutathione is an essential factor in protecting the pulmonary system from toxic insults [Potdar et al. 1997] and $\gamma$-glutamyl transferase expression can be detected in adult type II alveolar epithelial cells and Clara cells of the upper airway [Jean et al. 2003]. Both cell types need extensive antioxidant defences and require glutathione as a substrate for xenobiotic metabolism with particular focus on the upper airway since it is a primary site for deposition of inhaled particulate matter. Phenylethanolamine $\mathrm{N}$ methyltransferase is the terminal enzyme of the catecholamine biosynthesis pathway and normally methylates norepinephrine to convert this into the active form epinephrine. [Ji et al. 2005]. This enzyme has been reported to methylate the monoamine oxidase inhibitor Phenelzine [Yu et al. 1991], however methylation is generally a minor pathway of xenobiotic transformation [Jancova et al. 2012] so expression of PNMT is an interesting observation but may be of lower significance than GGT1.

The absence of glutathione-S-transferase $\mu 1$ (GSTM1) in both models is interesting since lack of GTSM expression has been linked to increased risk of developing adverse health effects following exposure to pro-oxidant air pollutants such as diesel exhaust and cigarette smoke [Peden, DB. 2005; Romieu et al. 2004]. GSTM1 is regulated by NRF2 which is expressed by both models, so its absence is difficult to explain. For the pBAE model, this could be cell line dependent and may correlate with occurrence of the so-called GSTM1 null phenotype reported in up to $62 \%$ of individuals of European descent [Geisler et al. 2001], however the undifferentiated iPSC line used to generate the iBAE model clearly expresses GSTM1, so this explanation is unlikely for the iPSC derived airway construct. Other glutathione-S-transferases (GSTM2 \& GTSM3) are expressed at comparable levels by both models.

The TempoSeq gene set contains a broad range of solute channel transporter proteins and the majority of these show comparable although not precisely equal expression levels between iBAE and pBAE. Notable exceptions include members of the glutamate transporter channel class (SLC17A6), sulphate transporter (SLC26A2), glucose transporters 14 and 3 (SLC2A14, SLC2A3) and choline transporter like protein 4 (SLC44A4). The impact of these proteins on the capability of either model to transport xenobiotic substances and their metabolites across the membranes of the cells that comprise the iBAE and pBAE models is not clear.

The primary focus of this study was to characterise the transcriptional responses of the two models to xenobiotic exposure and to gain insights into the induction of toxicity pathways at sub-lethal concentrations of the drugs as described. Analysis of the metabolites generated by each models would be the next step in understanding the toxicological response of each model in more detail but this was outside the scope of the current study. The TempoSeq data obtained indicate that the two models may be of sufficient similarity to allow use of the iBAE model as a tool for toxicological assessment that in mind, we exposed both models to 5 compounds with known lung toxicities and analysed transcriptomic 
changes using TempoSeq. Differentially expressed genes were assigned to known toxicity pathways and showed similar changes in expression levels between the iBAE and pBAE models after xenobiotic exposure. Molecules such as paraquat, Busulfan and benzo(a) pyrene activate pathways such as the aryl hydrocarbon response, p53, nRF2 And ATF4 in line with expectations while Amiodarone and Cerium oxide particles show repression of many pathways rather than statistically significant activation. This may be related to known effects of the substances. Amiodarone is reported to cause pulmonary fibrosis upon chronic exposure to the drug but iBAE and pBAE models cannot address this since they only comprise a pseudostratified airway epithelium and lack the stromal and vascular components that would normally contribute to fibrotic development. This latter observation highlights a key limitation of this study in that we can only use the models in their current forms to assess toxic response of the airway epithelium and a more comprehensive study would require not only a greater range of substances over a range of reported toxicities directed against the upper airway but also the inclusion of greater structural complexity to fully replicate the cellular architecture of the upper airway. In addition, the use of a "bulk" RNA sequencing approach on a complex mixture of cell types such as the iBAE and pBAE models does not quantify the toxicological responses of individual cell types therefore single cell RNA sequencing may be required in future studies to obtain these data.

\section{Declarations}

Availability of data and materials: The datasets supporting the conclusions of this article are included within the article and its supplementary files

Author contributions: Ivo Djidrovksi (ID): experimental design, data acquisition, data interpretation and manuscript preparation. Maria Georgiou (MG): data acquisition and interpretation. Elena Tasinato (ET): data acquisition. Martin $\mathrm{O}$ Leonard (MOL): experimental design and data interpretation. Jelle van den Bor (JVB): experimental design and data acquisition. Majlinda Lako (ML): experimental design, funding and manuscript preparation. Lyle Armstrong (LA): exeprimental design, data interpretation, funding and manuscript preparation.

Acknowledgements: Financial support from the Marie Skłodowska-Curie Action - Innovative Training Network, grant number 721975 (IN3) and grant funding from the Biotechnology and Biosciences Research Council (BBSRC) (grant reference BB/V01126X/1) held by Majlinda Lako and colleagues.

Funding: Financial support from the Marie Skłodowska-Curie Action - Innovative Training Network, grant number 721975 (IN3) and grant funding from the Biotechnology and Biosciences Research Council (BBSRC) (grant reference BB/V01126X/1) held by Majlinda Lako and colleagues.

Conflicts of interest: The authors declare no competing interests in this publication

Ethics Approval: Not applicable

Consent to participate: Not applicable 
Consent for publication: All authors have given consent to publish this manuscript

Availability of data: The datasets supporting the conclusions of this article are included within the article and its supplementary files

Code availability: Not applicable

\section{References}

1. Acosta-Alvear, D., Zhou, Y., Blais ,A., Tsikitis, M., Lents, NH., Arias, C, Lennon, CJ., Kluger, Y., Dynlacht BD. (2007) XBP1 controls diverse cell type- and condition-specific transcriptional regulatory networks. Mol Cell 27(1):53-66

2. Anderson, CM., Stahl, A. (2013) SLC27 fatty acid transport proteins. Mol Aspects Med. 34(2-3): 516528

3. Aufderheide, M., Scheffler, S., Ito, S., Ishikawa, S. \& Emuraa, M. (2015) Ciliatoxicity in human primary bronchiolar epithelial cells afterrepeated exposure at the air-liquid interface with native mainstreamsmoke of K3R4F cigarettes with and without charcoal filter. Exp. Toxicol. Pathol. 67: 407-411

4. Baritussio, A., Marzini, S., Agostini, M., Alberti, A., Cimenti, CC., Bruttomesso, D., Manzato, E., Quagliano, D., Pettenazzo, A. (2001) Amiodarone inhibits lung degradation of SP-A and perturbs the distribution of lysosomal enzymes. Am J Physiol Lung Cell Mol Physio/ 281: L1189-L1199.

5. B'chir, W., Maurin, AC., Carraro, V., Averous, J., Jousse, C., Muranishi, Y., Parry, L., Stepien, G., Fafournoux, P., Bruhat, A. (2013) The elF2a/ATF4 pathway is essential for stress-induced autophagy gene expression. Nucleic Acids Res 41(16):7683-99

6. Blanco-Ayala T, Anderica-Romero AC, Pedraza-Chaverri J. (2014) New insights into antioxidant strategies against paraquat toxicity. Free Radic Res 48(6):623-40

7. Boei, J. J. W. A. et al. (2017) Xenobiotic metabolism in differentiated human bronchial epithelial cells. Arch. Toxicol. 91, 2093-2105.

8. Brown D, Alexander R, MarchR, Morgan P, Satterthwaite G, Pangalos MN (2014) · Lessons learned from the fate of AstraZeneca's drug pipeline: a five-dimensional framework. Nat Rev Drug Discov 13(6):419-31

9. Bushel, P. R., Paules, R. S. \& Auerbach, S. S. (2018) A Comparison of the TempO-Seq S1500+ Platform to RNA-Seq and Microarray Using Rat Liver Mode of Action Samples. Front. Genet. 9, 485.

10. Buskin, A., Zhu, L., Chichagova, V. et al. Disrupted alternative splicing for genes implicated in splicing and ciliogenesis causes PRPF31 retinitis pigmentosa. Nat Commun 9, 4234 (2018)]

11. Carlson, GP. (2008) Critical appraisal of the expression of cytochrome P450 enzymes in human lung and evaluation of the possibility that such expression provides evidence of potential styrene tumorigenicity in humans. Toxicology 254(1-2): 1-10 
12. Chen, YB., Coughlin, E., Kennedy, KF., Alyea, EP., Armand, P., Attar, EC., Ballen, KK., Cutler, C., Dey, BR., Koreth, J., McAfee, SL., Spitzer, TR., Antin, JH., Soiffer, RJ., Ho, VT. (2013) Busulfan dose intensity and outcomes in reduced-intensity allogeneic peripheral blood stem cell transplantation for myelodysplastic syndrome or acute myeloid leukemia. Biol Blood Marrow Transplant 19(6):981-7

13. Courcot,E., Leclerc, J., Lafitte, JJ., Mensier, E., Jaillard, S., Gosset, P., Shirali, P., Pottier, N., Broly, G., LoGuidice, J. (2012). Xenobiotic metabolism and disposition in human lung cell models: comparison with in vivo expression profiles. Drug Metab Dispos. 40(10):1953-65

14. Danielson, PB. (2002) The cytochrome P450 superfamily: biochemistry, evolution and drug metabolism in humans. Curr Drug Metab 3(6):561-97

15. de Jong, P. M. et al (1994). Ciliogenesis in human bronchial epithelial cells cultured at the air-liquid interface. Am. J. Respir. Cell Mol. Biol. 10: 271-277

16. Djidrovski, I, Georgiou M, Hughes GL, Patterson El, Casa-Sanchez A, Pennington SH, Biagini G, MoysMolina M. van den Bor J, Smit M, Chung G, Lako M, Armstrong L. (2021) SARS-CoV-2 infects an upper airway model derived from induced pluripotent stem cells. Stem cells 39(1): 1319-1321

17. Gassmann, K., Abel, J., Bothe, H., Haarmann-Stemmann, T., Merk, HF., Quasthoff, KN., Dino Rockel, TD., Schreiber, T., and Fritsche E. (2010) Species-Specific Differential AhR Expression Protects Human Neural Progenitor Cells against Developmental Neurotoxicity of PAHs. Environ Health Perspect. 118(11): 1571-1577.

18. Geisler, SA., Olshan, AF. (2001) GSTM1, GSTT1, and the risk of squamous cell carcinoma of the head and neck: a mini-HuGE review. Am J Epidemiol. 154(2):95-105

19. Gonzalez-Polo RA, Rodriguez-Martin A, Moran JM, Niso M, Soler G, Fuentes JM. (2004) Paraquatinduced apoptotic cell death in cerebellar granule cells. Brain Res 1011(2):170-6

20. Göttlicher, M. (1999) Chapter 10 -Receptor Toxicology.in Toxicology, ISBN 978-0-12-473270-4

21. Guerrina, N., Traboulsi, H., Eidelman, DH., Baglole, CJ. (2018) The Aryl Hydrocarbon Receptor and the Maintenance of Lung Health. Int J Mol Sci. 19(12): 3882

22. Gutiérrez-Aguilar, M., CP. (2013) Physiological and pathological roles of mitochondrial SLC25 carriers. Biochem J. 454(3): 371-386.

23. Halliwell, WH(1997) Cationic amphiphilic drug-induced phospholipidosis. Toxicol Pathol 25(1):53-60

24. Harding, HP., Novoa, I., Zhang, Y., Zeng,H., Wek,R., Schapira, M., Ron, D. (2000) Regulated translation initiation controls stress-induced gene expression in mammalian cells. Mol Cell 6(5): 1099-108

25. Harris, SL. \& Levine, AJ. (2005) The p53 pathway: positive and negative feedback loops. Oncogene 24: $2899-2908$

26. Hayashi, A., Suzuki, H., Itoh, K., Yamamoto, M., Sugiyama Y. (2003). Transcription factor Nrf2 is required for the constitutive and inducible expression of multidrug resistance-associated protein 1 in mouse embryo fibroblasts. Biochem. Biophys. Res. Commun. 310:824-29

27. Hayden, MS., S. (2008) Shared principles in NF-kappaB signaling. Cell 132(3):344-62 
28. Hediger, MA., Romero, MF., Peng ,JB., Rolfs, A., Takanaga, H., Bruford, EA. (2004) The ABCs of solute carriers: physiological, pathological and therapeutic implications of human membrane transport proteins Introduction. Pflügers Archiv. 447 (5): 465-8.

29. Heneghan, JF., Akhavein, A., Salas, MJ., Shmukler, BE., Karniski, LP., Vandorpe, DH., Alper, SL. (2010) Regulated transport of sulfate and oxalate by SLC26A2/DTDST. Am J Physio/ Cell Physiol. 298(6): C1363-C1375.

30. House, J. S. et al. (2017) A Pipeline for High-Throughput Concentration Response Modeling of Gene Expression for Toxicogenomics. Front. Genet. 8: 168

31. Hu, H., Tian, M., Ding C., Yu, S. (2019) The C/EBP Homologous Protein (CHOP) Transcription Factor Functions in Endoplasmic Reticulum Stress-Induced Apoptosis and Microbial Infection. Front Immuno/ 9:3083

32. Huang, S., Wiszniewski, L., Constant, S., Roggen, E. (2013) Potential of in vitro reconstituted 3D human airway epithelia (MucilAir ${ }^{\text {TM }}$ ) to assess respiratory sensitizers. Toxicol In Vitro. 27(3):1151-6

33. Jancova, P., Siller, M. (2012) Phase II Dru Metabolism: https://www.intechopen.com/books/topicson-drug metabolism/phase-ii-metabolism

34. Jean, JC., Liu, Y., Joyce-Brady, M. (2003) The importance of gamma-glutamyl transferase in lung glutathione homeostasis and antioxidant defense. BioFactors 17: 161-173

35. Ji Y. Salavaggione O. Wang L. Adjei A. A. Eckloff B. Wieben E. D. Weinshilboum R. M. (2005) Human phenylethanolamine $\mathrm{N}$-methyltransferase pharmacogenomics: gene re-sequencing and functional genomics. Journal of Neurochemisty 95(6): 1766-1776

36. Jin, Y., Feng, M., Ma, W., Wei, Y., Qi, G., Luo, J., Xu, L., Li, X., Li, C., Wang, Y., Li, D., Chen ' J., Zhao, Y., Hou, Y., Zhao, Q., Jiang, L., Xie' M, Zheng, Y., Yu, D. (2021) A toxicity pathway-oriented approach to develop adverse outcome pathway: AHR activation as a case study. Environ Pollut 268(Pt B):115733

37. Kanai, Y., Clémençon, B., Simonin, A., Michele Leuenberger,M., Lochner, M., Weisstanner, LM., Hediger,MA. (2013) The SLC1 high-affinity glutamate and neutral amino acid transporter family. Molecular Aspects of Medicine. 34(2-3): 108-120

38. Kensler, TW., Wakabayashi, N., and Biswal, S. C (2007) Cell Survival Responses to Environmental Stresses Via the Keap1-Nrf2-ARE Pathway. Annu. Rev. Pharmacol. Toxicol. 47:89-116

39. Kim, JH., Sherman, ME., Curriero, FC., Guengerich, FP., Strickland, PT., Sutter, TR. (2004) Expression of cytochromes P450 1A1 and 1B1 in human lung from smokers, non-smokers and ex-smokers. Toxicol Appl Pharmacol. 199: 210-219

40. Kutmon, M., van lersel, MP., Bohler, A., Kelder, T., Nunes, N., Pico, AR., Evelo, CT. (2015) PathVisio 3: An Extendable Pathway Analysis Toolbox PLoS Comput Biol. 11(2): e1004085

41. Kwak, MK., Wakabayashi, N., Greenlaw, JL., Yamamoto, M., Kensler, TW. (2003). Antioxidants enhance mammalian proteasome expression through the Keap1- Nrf2 signaling pathway. Mol. Cell. Biol. 23:8786-94 
42. Laity, JH., Andrews, GK. (2007) Understanding the mechanisms of zinc-sensing by metal-response element binding transcription factor-1 (MTF-1). Arch Biochem Biophys 463(2):201-10.

43. Lawrence, L. (2009) The Nuclear Factor NF-KB Pathway in Inflammation. Cold Spring Harb Perspect Biol. 1(6): a001651.

44. Lee, AH., Iwakoshi, NN., Glimcher, LH., (2003) XBP-1 regulates a subset of endoplasmic reticulum resident chaperone genes in the unfolded protein response. Molecular and Cellular Biology. 23(21):7448-7459

45. Lizák, B., Szarka, A., Kim, Y., Choi, KY., Németh, CE., Marcolongo, P., Benedetti, A., Bánhegyi, G., Margittai, E. (2019) Glucose Transport and Transporters in the Endomembranes. Int J Mol Sci. 20(23): 5898.

46. Love, M. I., Huber, W. \& Anders, S. (2014) Moderated estimation of fold change and dispersion for RNA-seq data with DESeq2. Genome Biol. 15, 550 (2014).

47. Masoud, GN., Li, W. HIF-1 a pathway: role, regulation and intervention for cancer therapy. Acta Pharm Sin B 2015 Sep;5(5):378-89

48. Mav, D. et al. (2018) A hybrid gene selection approach to create the $\mathrm{S} 1500+$ targeted gene sets for use in high-throughput transcriptomics. PLoS One 13, e0191105

49. Michalik, L., Auwerx, J., Berger, JP., Chatterjee, VK., Glass, CK., Gonzalez, FJ. et-al (2006). International Union of Pharmacology. LXI. Peroxisome proliferator-activated receptors. Pharmacological Reviews. 58(4): 726-41

50. Moinova, HR., Mulcahy, RT. (1999). Up-regulation of the human gammaglutamylcysteine synthetase regulatory subunit gene involves binding of Nrf-2 to an electrophile responsive element. Biochem. Biophys. Res. Commun. 261:661- 68

51. Mottis, A., Jovaisaite, V., Auwerx, J. (2014) The mitochondrial unfolded protein response in mammalian physiology. Mamm Genome. 25(0): 424-433

52. Nabokina, SM., Ramos MB., Valle, JE., Said, HM. (2015) Regulation of basal promoter activity of the human thiamine pyrophosphate transporter SLC44A4 in human intestinal epithelial cells. Am J Physiol Cell Physiol. 308(9): C750-C757

53. Oliner, H., Schwartz, R., Rubio, F., Dameshek, W. (1961) Interstitial pulmonary fibrosis following busulfan therapy. Am J Med 31:134-9

54. Olsson, I. A. S., da Silva, S. P., Townend, D. \& Sandøe, P. Protecting animals and enabling research in the European Union: An overview of development and implementation of directive 2010/63/EU. ILAR J. 57, 347-357 (2016).

55. Potdar, PD Andrews, KL, Nettesheim, P, Ostrowski LE. Expression and regulation of gamma-glutamyl transpeptidase-related enzyme in tracheal cells. Am J Physiol. 1997 Nov;273(5):L1082-9

56. Pakos-Zebrucka, K., Koryga, I., Mnich, K., Ljujic, M., Samal, A., Gorman, AM. (2016) The integrated stress response. EMBO Rep 17:1374-1395 
57. Peden, DB. (2005) The epidemiology and genetics of asthma risk associated with air pollution. $J$ Allergy Clin Immunol. 115(2):213-219

58. Pezzulo, A. A. et al. (2011) The air-liquid interface and use of primary cell cultures are important to recapitulate the transcriptional profile of in vivo airway epithelia. Am. J. Physiol. - Lung Cell. Mol. Physiol. 300: 25-31

59. Prestera, T., Talalay, P., Alam, J., Ahn, Yl., Lee, PJ., Choi AM. (1995). Parallel induction of heme oxygenase- 1 and chemoprotective phase 2 enzymes by electrophiles and antioxidants: regulation by upstream antioxidant-responsive elements (ARE). Mol. Med. 1: 827-37

60. Primiano, T., Li, Y., Kensler, TW., Trush, MA., Sutter, TR. (1998). Identification of dithiolethioneinducible gene-1 as a leukotriene B4 12-hydroxydehydrogenase: implications for chemoprevention. Carcinogenesis 19:999-1005

61. Romieu, I., Sienra-Monge, JJ., Ramirez-Aguilar, M., Moreno-Macias, H., Reyes-Ruiz, NI., Estela del RioNavarro, B., Hernandez-Avila, M., London, SJ. (2004) Genetic polymorphism of GSTM1 and antioxidant supplementation influence lung function in relation to ozone exposure in asthmatic children in Mexico City. Thorax 59:8-10

62. Ross, WE., Block, ER., Chang, RY. (1979) Paraquat-induced DNA damage in mammalian cells. Biochem Biophys Res Commun. 91(4):1302-8

63. Sexton, KJ., Burube KA. (2008) An in vitro approach to assess the toxicity of inhaled tobacco smoke components: nicotine, cadmium, formaldehyde and urethane. Toxicology 244 (1): 66-76.

64. Shimada, T. (2006) Xenobiotic-metabolizing enzymes involved in activation and detoxification of carcinogenic polycyclic aromatic hydrocarbons. Drug Metab Pharmacokinet 21:257-276

65. Shimada, T., Yamasaki, H., Mimura, M., Wakamiya, N., Ueng, YF., Guengerich, FP., Inui, Y. (1996) Characterisation of microsomal cytochrome P450 enzymes involved in the oxidation of xenobiotic chemicals in human fetal livers and adult lung. Drug Metab Dispos 24: 515-522

66. Sykes, A. V., Baptista, F. D., Gonçalves, R. A. \& Andrade, J. P. (2012) Directive 2010/63/EU on animal welfare: a review on the existing scientific knowledge and implications in cephalopod aquaculture research. Rev. Aquac. 4, 142-162

67. Wang, S., Chanock, S., Tang, D., Li, Z., Jedrychowski, W., Perera, FP. (2005) Assessment of Interactions between PAH Exposure and Genetic Polymorphisms on PAH-DNA Adducts in African American, Dominican, and Caucasian Mothers and Newborns. Cancer Epidemiol Biomarkers Prev 14(3):709-14

68. Willey, JC. , Coy, EL. , Frampton, MW. , Torres, A , Apostolakos, MJ , Hoehn, G. , Schuermann, WH. , Thilly, WG. , Olson, DE. , Hammersley, JR. , Crespi, CL. , Utell, MJ. (1997) Quantitative RT-PCR Measurement of Cytochromes p450 1A1, 1B1, and 2B7, Microsomal Epoxide Hydrolase, and NADPH Oxidoreductase Expression in Lung Cells of Smokers and Nonsmokers. Am J Respir Cell Mol Biol. 17(1):114-24

69. Xu, Y., Wang, Y.,Besnard, V.,Ikegami, M.,Wert, SE.,Heffner, C.,Murray, SA.,Donahue, LR., Whitsett, JA. (2012) Transcriptional Programs Controlling Perinatal Lung Maturation. PLoS One. 7(8): e37046. 
70. Yu, PH., Davis, BA., Durden, DA. (1991) Enzymatic N-methylation of phenelzine catalyzed by methyltransferases from adrenal and other tissues. Drug Metab Dispos 19(4):830-4.

71. Zhang, J., Fen Wang, Y. \& Prakash, C. (2006) Xenobiotic-Metabolizing Enzymes in Human Lung. Curr.Drug Metab. 7, 939-948

\section{Supplementary Tables}

Supplementary Table 1 is not available with this version.

\section{Figures}

A)

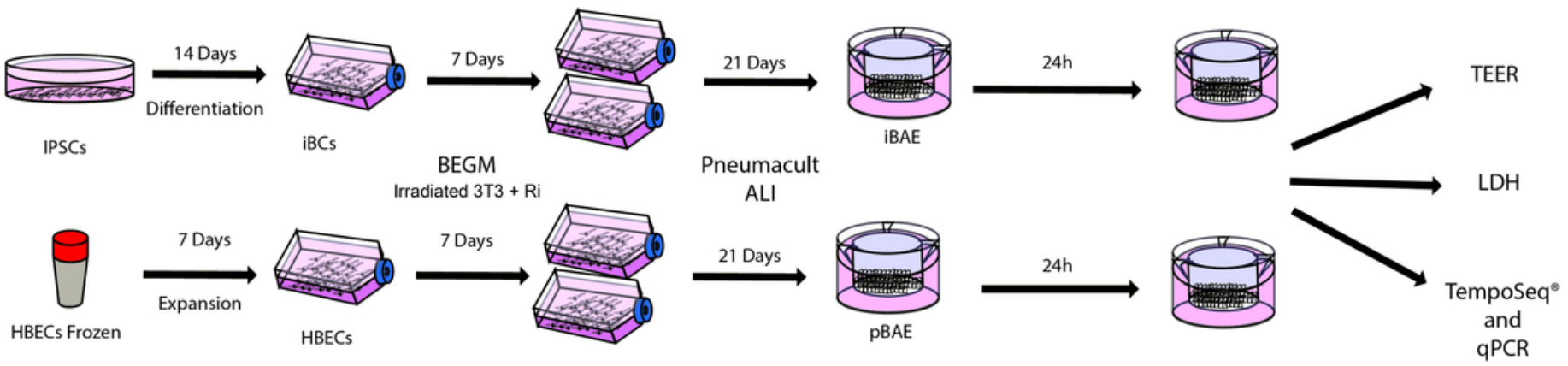

B)



iBAE day 21 ALI

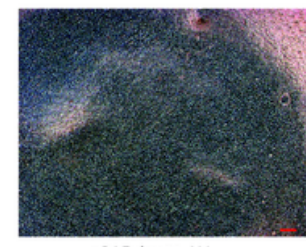

PBAE day 21 ALI

E)
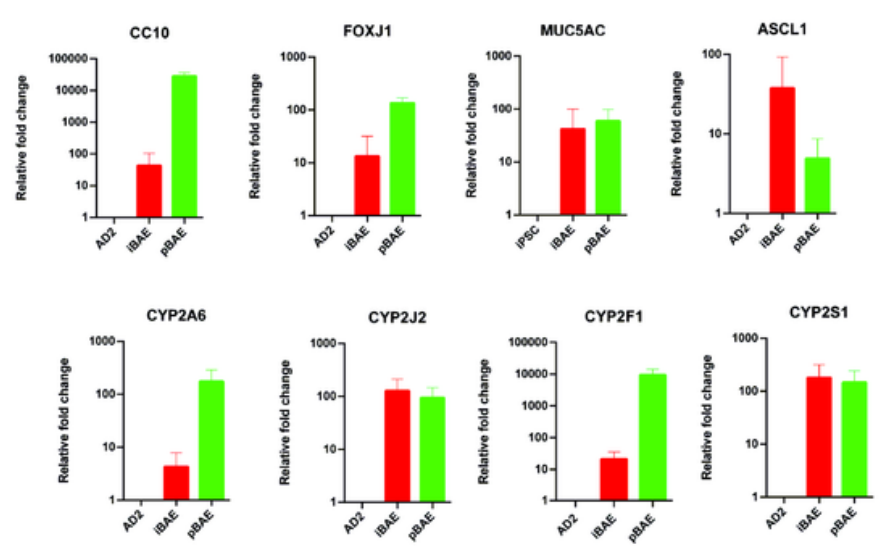

C)
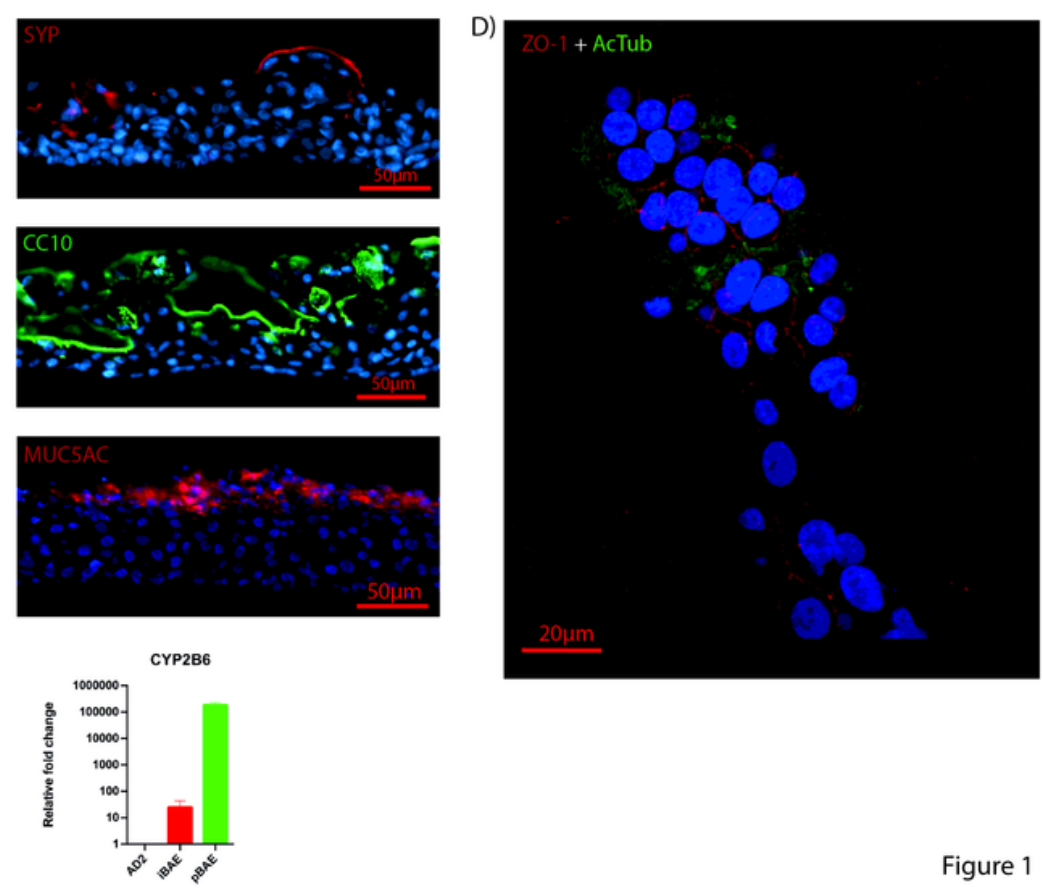

Figure 1

Figure 1

. (A) Schematic protocol for generation of upper airway basal like cells from iPSCs using the protocol detailed in the materials and methods section followed by differentiation at an ALI to produce pseudostratified airway epithelial constructs from both iPSC derived and primary human bronchial epithelial cells (HBECs). (B) Brightfield images of the iPSC derived (iBAE) and HBEC derived (pBAE) constructs after 21 days differentiation at an air-liquid interface. Scale bars (red) in B represent 50 $\mu$ m. 
Representative images from 6 experiments in both cell lines are shown). (C) Characterisation of the pseudostratified epithelia derived from primary, and iPSC generated basal cells indicates the presence of pulmonary neuroendocrine cells: Synaptophysin expression, club cell protein (CC10) expressing cells and Mucin 5AC expressing goblet cells. (D) The apical surface of the pseudostratified epithelium comprises ciliated epithelial cells capable of forming tight junction indicated by the presence of ZO-1 and cilia indicated by the presence of acetylated tubulin (AcTub). All scale bars for C-D as shown. Representative images from 6 experiments in both cell lines are shown. (E) Relative expression of marker genes of upper airway epithelium cell types in IBAE and pBAE constructs compared to undifferentiated WT1 iPSC line (CC10 (club cells), FOXJ1 (ciliated airway epithelial cells), MUC5AC (goblet cells) and ASCL1 (pulmonary neuroendocrine cells)) measured by quantitative RT-PCR. Data are shown as mean+/SEM, $n=3$. and relative expression of cytochrome p450 enzyme encoding genes in iBAE and pBAE constructs compared to undifferentiated WT1 iPSC line. Data are shown as mean+/SEM, $n=3$.

A)

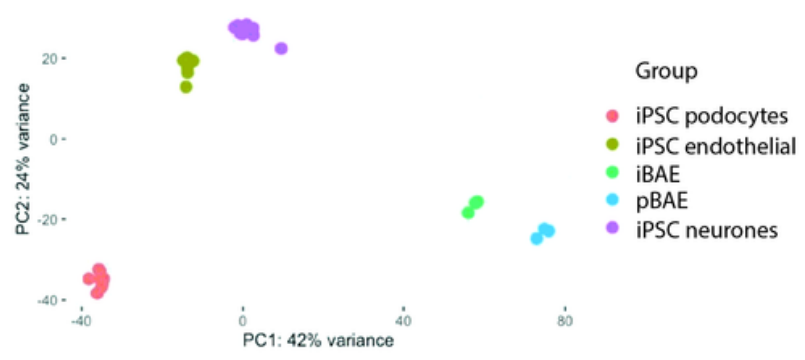

B)

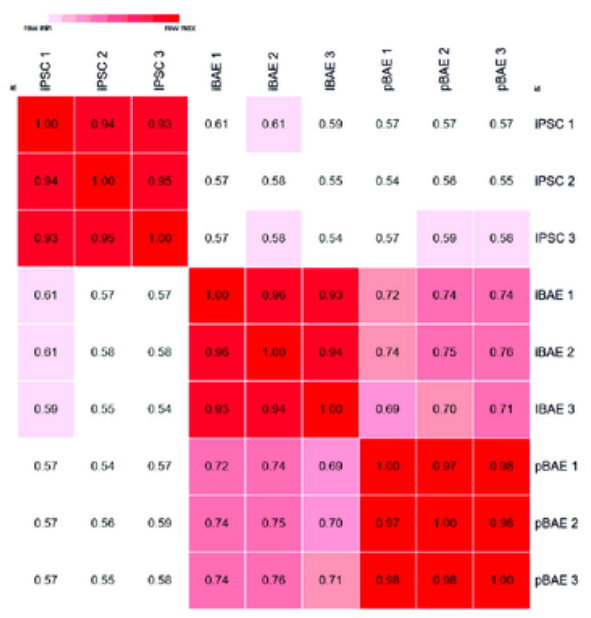

C)

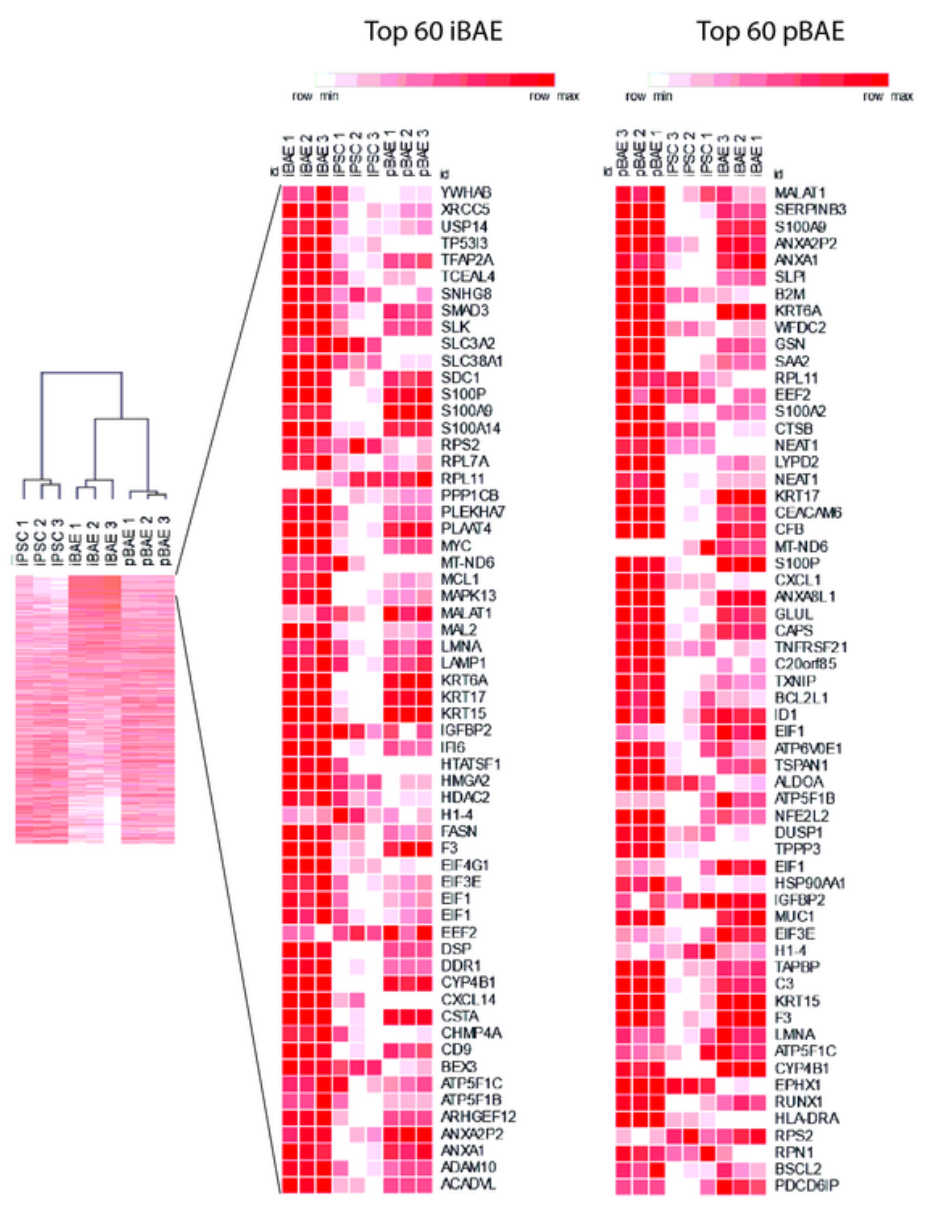

Figure 2

\section{Figure 2}

TemposeqTM transcriptomic analysis of the relative expression of genes represented on the TemposeqTM gene set between $\mathrm{BBAE}$ and pBAE models with comparison to undifferentiated iPSCs. (A) 
Principal component analysis shows the expression of genes on the TemposeqTM data set clusters the iBAE and pBAE constructs together while distinguishing them from other iPSC derived cell types generated by the IN3 consortium with lower variance between the airway constructs samples $(n=3)$ subjected to TemposeqTM analysis. (B) Pearson correlation indicated low variability between expression levels of TemposeqTM genes in $\mathrm{BAAE}$ and $\mathrm{pBAE}$ constructs suggesting their similarity. Comparison of these models was extended to 60 highest expressed genes present in both models shown in the heatmap (C) of log2 fold changes generated using the software package Pheatmap. The heatmaps are arranged as follows: from left to right; three columns represent log2 fold changes for genes in triplicate for the iBAE model, three columns represent expression levels of the same genes in undifferentiated iPSC while three remaining columns represent three replicates of the pBAE model. Data presented are derived from triplicate repeats of TemposeqTM analysis using biological replicates of iBAE and pBAE constructs.

A)

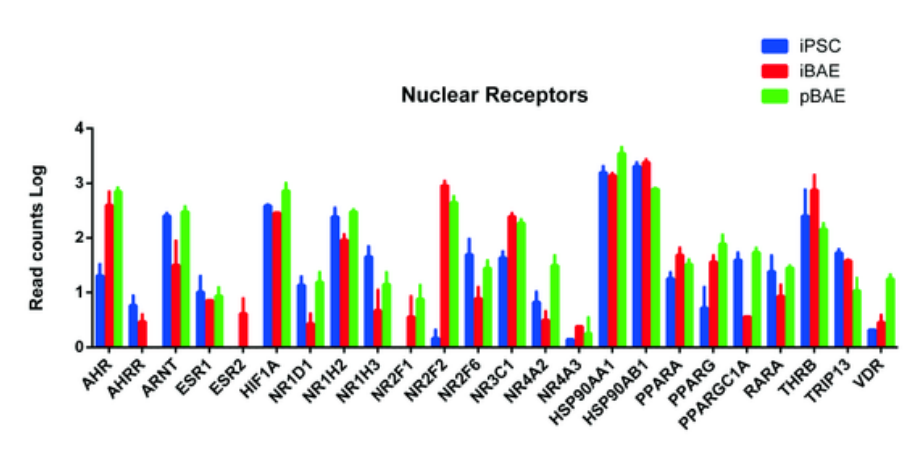

B)

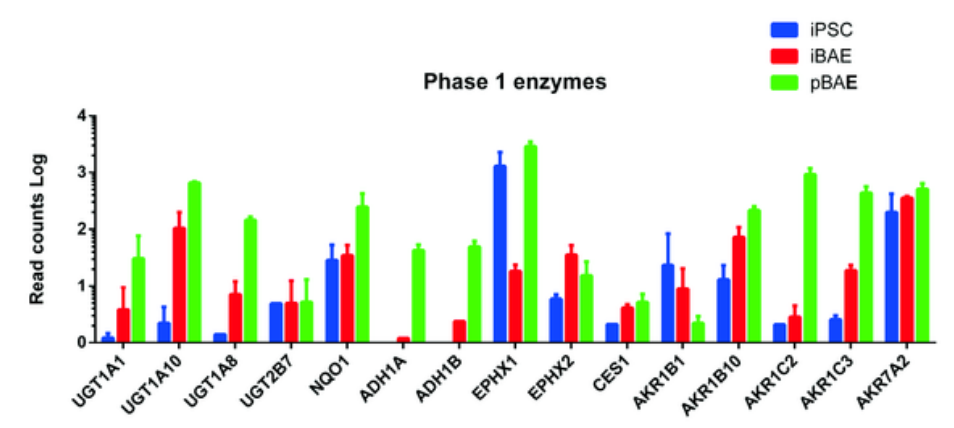

C)

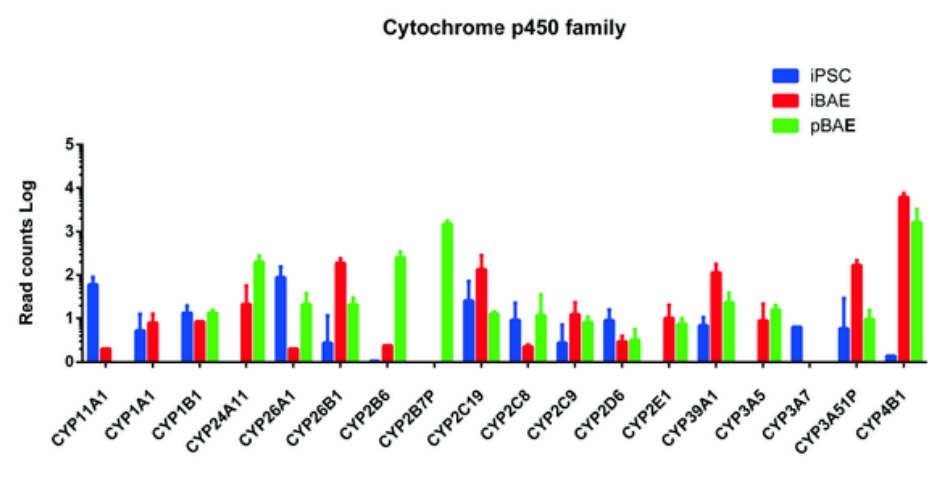

D)

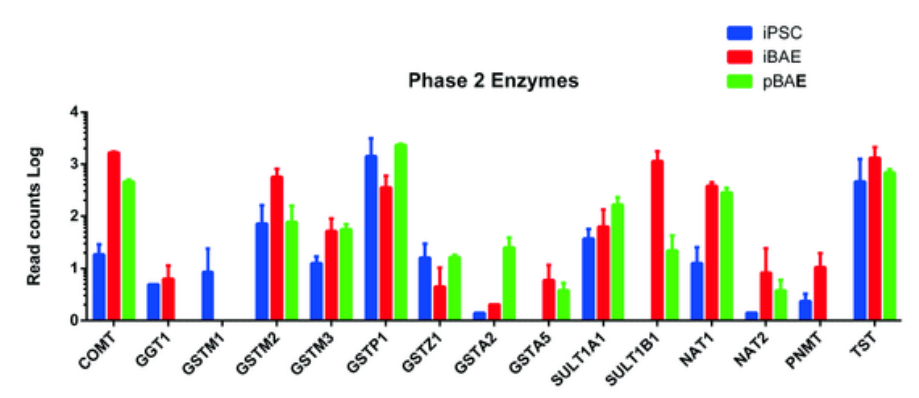

Figure 3

\section{Figure 3}

The iBAE and pBAE models show similar expression of (A) nuclear receptor encoding genes, (B) genes encoding enzymes involved in phase 1 metabolic transformation, (C) genes encoding cytochrome P450 family enzymes and (D) genes encoding enzymes involved in phase 2 metabolism (those which are present in the TemposeqTM data set). Data presented are derived from triplicate repeats of TemposeqTM analysis using biological replicates of iBAE and pBAE constructs. 

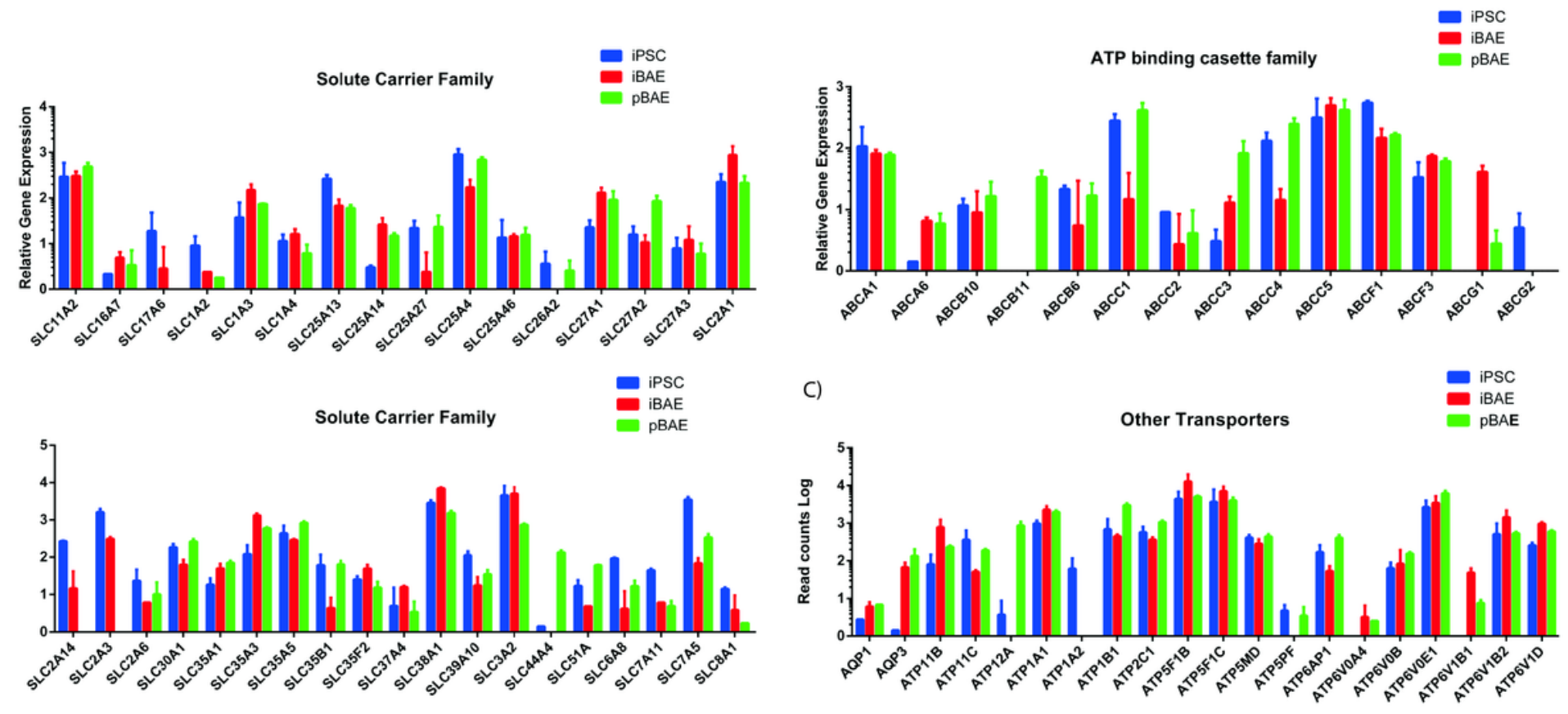

Figure 4

\section{Figure 4}

The iBAE and pBAE models show similar expression of (A, B). Genes encoding solute carrier proteins (C) genes encoding ATP binding cassette family proteins and (D) other transporter proteins all classes of which are involved in the removal of xenobiotic molecules or their metabolites from cells of the upper airway epithelium. Data presented are derived from triplicate repeats of TemposeqTM analysis using biological replicates of iBAE and pBAE constructs. 


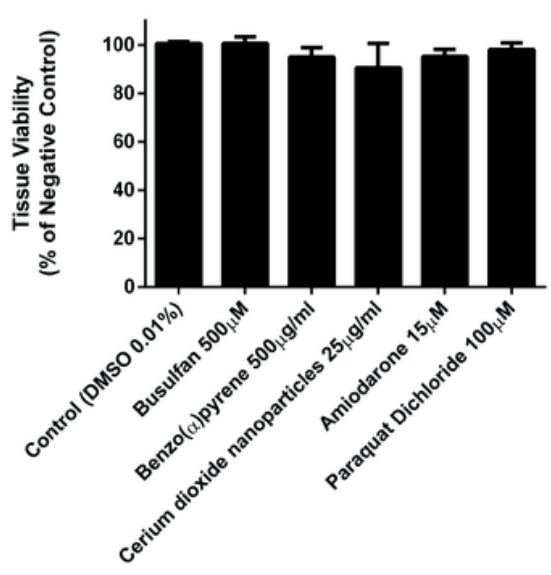

iBAE

B)

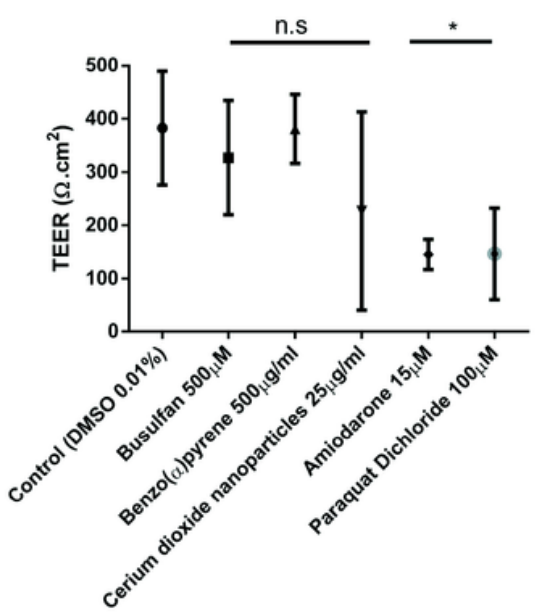

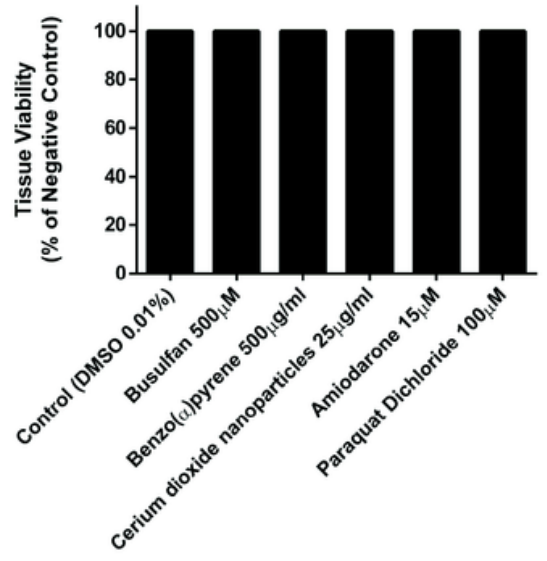

pBAE

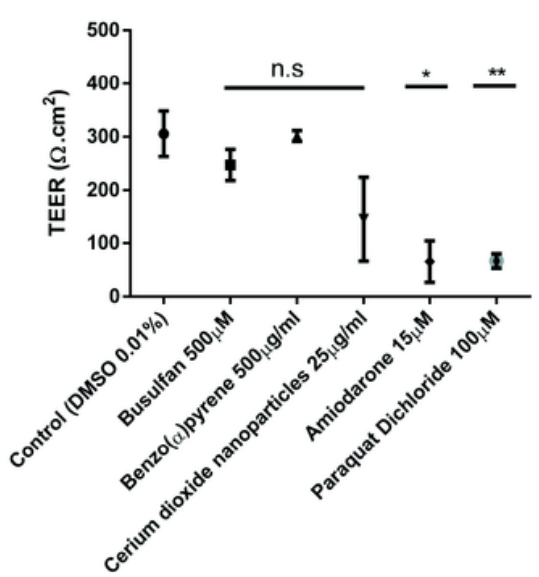

Figure 5

Figure 5

(A) Response to the iBAE and pBAE upper airway constructs to Busulfan, Benzo(a) pyrene, cerium dioxide nanoparticles, amiodarone and paraquat dichloride (24-hour exposure) measured by the production of lactate dehydrogenase (LDH). Data are presented as mean+/SEM, $n=3$ and expressed as a percentage viability compared to negative control iBAE and pBAE constructs not exposed to either vehicle (DMSO) or test compounds. (B) Response of TEER to vehicle only (DMSO) and test compounds at the concentrations stated in the figure (24-hour exposure). TEER measured in Ohms/cm2, Data presented as mean+/SEM, $n=3$. 
A)

Cerium Nanoparticles

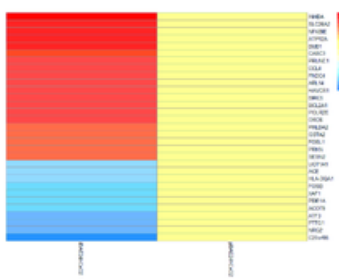

B)

iBAE

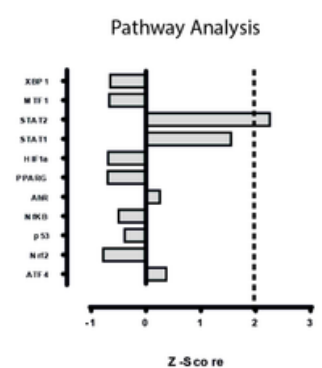

Pathway Analysis

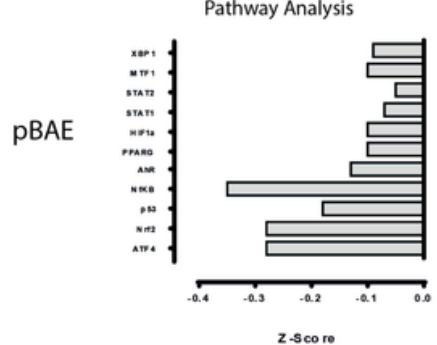

Amiodarone

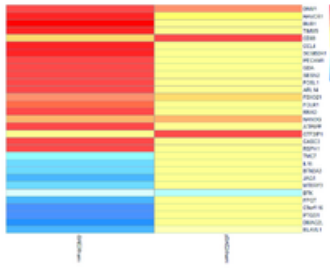

Pathway Analysis

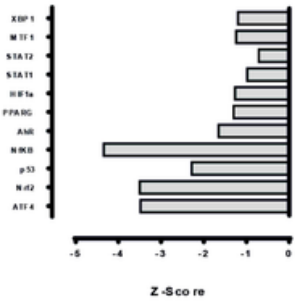

Pathway Analysis

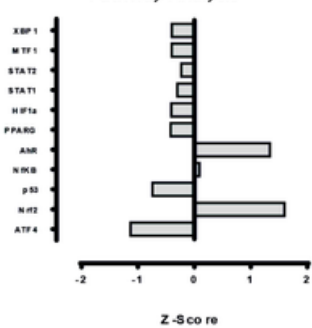

Busulfan

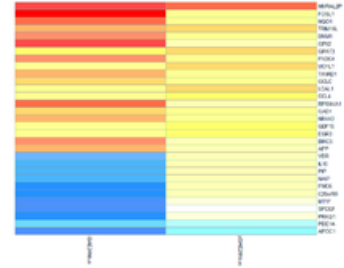

Pathway Analysis

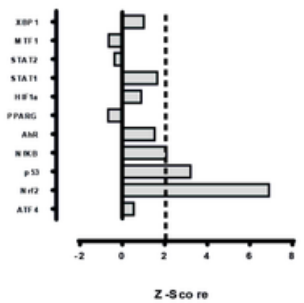

Pathway Analysis

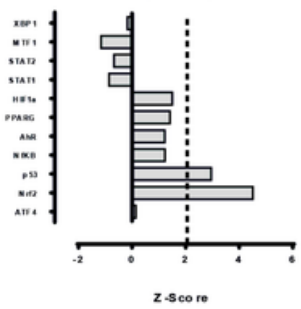

Paraquat Dichloride

Benzo(a)pyrene
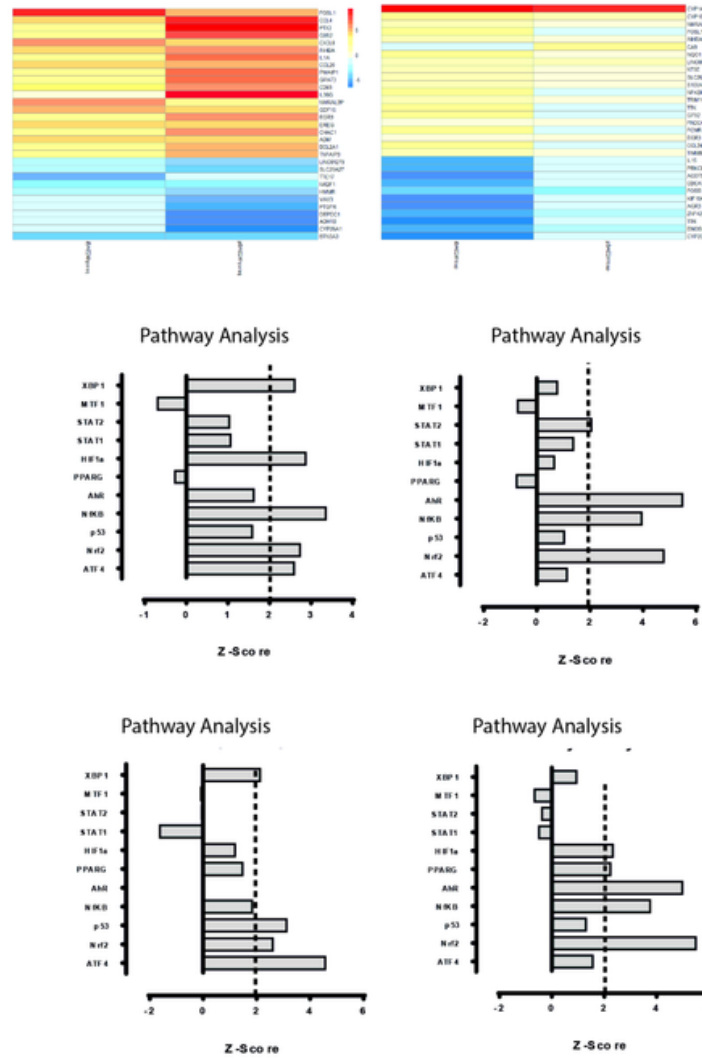

Figure 6

Figure 6

(A) Response of the iBAE and pBAE upper airway constructs to Busulfan, Benzo(a) pyrene, cerium dioxide nanoparticles, amiodarone and paraquat dichloride analysed by numbers of genes from the TemposeqTM data set undergoing statistically significant log fold change after 24-hour exposure relative to untreated controls. (B) Analysis of log2 fold changes occurring in genes identified from by TemposeqTM analysis that are participants in the major toxicity associated pathways (ATF4, NRF2, NFkB, AhR, PPARG, HIF1a, STAT1/2, MTF1 and XBP1) by quantification of Z-score (see supplementary table 1 for method of Z-score calculation). Data presented are derived from triplicate repeats of TemposeqTM analysis using biological replicates of iBAE and pBAE constructs.

\section{Supplementary Files}

This is a list of supplementary files associated with this preprint. Click to download.

- figureS1final002copy.tif 